\title{
Finger Replacement Method for RAKE Receivers in the Soft Handover Region
}

\author{
Seyeong Choi, Member, IEEE, Mohamed-Slim Alouini, Senior Member, IEEE, \\ Khalid A. Qaraqe, Senior Member, IEEE, and Hong-Chuan Yang, Senior Member, IEEE
}

\begin{abstract}
We propose and analyze a new finger replacement technique that is applicable for RAKE receivers in the soft handover (SHO) region. More specifically, the receiver uses in the SHO region by default the strongest paths from the serving base station (BS) and only when the combined signal-to-noise ratio falls below a certain pre-determined threshold, the receiver uses more resolvable paths from the target BS to improve the performance. Instead of changing the configuration for all fingers, the receiver just compares the sum of the weakest paths out of the currently connected paths from the serving BS with the sum of the strongest paths from the target $B S$ and selects the better group. Using accurate statistical analysis, we investigate in this letter the tradeoff between error performance, average number of required path comparisons, and SHO overhead offered by this newly proposed scheme.
\end{abstract}

Index Terms-Fading channels, diversity techniques, RAKE receivers, handover, performance analysis.

\section{INTRODUCTION}

$\mathbf{T}$ O mitigate the effect of multi-path fading, RAKE receivers are used in conjunction with wideband systems such as wideband code division multiple access (WCDMA) and ultra wideband (UWB) systems. These receivers combine the resolvable paths in order to increase the overall signal-tonoise ratio (SNR) and lower the probability of deep fades [1, Section 9.5.1]. In the handover (HO) region, the number of available resolvable paths can be quite large since they come from the serving base station (BS) and the target BS. As such, it is of interest to develop diversity combining schemes that maintain low complexity and low processing power consumption for mobile units while using a minimal amount of additional network resources. Although many of researchers have been dedicated to the study of this finger assignment issue, to the best knowledge of the authors, detailed investigation on diversity combining techniques which can distinguish the resolvable paths coming from the serving BS and those from the target BS has seldom been considered before.

Manuscript received November 5, 2006; revised February 24, 2007 and July 4, 2007; accepted August 29 2007. The associate editor coordinating the review of this paper and approving it for publication was $\mathrm{H}$. $\mathrm{H}$. Chen. This work was supported in part by the Qatar Foundation for Education, Science, and Community Development, Qatar, in part by Qatar Telecom, Qatar, and in part by a Discovery Grant from NSERC, Canada.

S. Choi, M.-S. Alouini, and K. A. Qaraqe are with the Department of Electrical Engineering, Texas A\&M University at Qatar, PO Box 23874, Education City, Doha, Qatar (e-mail: \{seyeong.choi, alouini, khalid.qaraqe\}@qatar.tamu.edu).

H.-C. Yang is with the Department of Electrical and Computer Engineering, University of Victoria, BC, V8W 3P6, Canada (e-mail: hyang@ece.uvic.ca). Digital Object Identifier 10.1109/TWC.2008.060902.
Recently, by considering macroscopic diversity schemes, the authors proposed and analyzed a new finger reassignment scheme that maintains a low complexity and reduces the soft $\mathrm{HO}$ (SHO) overhead [2]. The main idea behind [2] is that, in the SHO region, whenever the received signal is of unsatisfactory quality, the receiver scans the additional resolvable paths from the target BS and selects the strongest paths among the total available paths from both the serving and the target BSs. It has been shown that this scheme can reduce the unnecessary path estimations and the SHO overhead compared to the conventional generalized selection combining (GSC) scheme [3]-[5] which always uses $L_{c} /\left(L+L_{a}\right)$-GSC in the SHO region where $L_{c}$ is the number of fingers, $L$ and $L_{a}$ are the total resolvable paths from the serving and the target BSs, respectively.

In this letter, we propose an alternative finger combining scheme that is also applicable to the SHO region and further reduces the $\mathrm{SHO}$ overhead at the expense of a certain degradation in performance (which we will accurately quantify in the body of this letter). For convenience sake, we call this alternative scheme in this letter as a Block Change scheme and the scheme in [2] as a Full GSC scheme. With the Block Change scheme, like the Full GSC scheme when the output SNR falls below the target SNR, the receiver scans the additional $L_{a}$ resolvable paths from the target BS while unlike the Full GSC scheme, the receiver compares the sum of the SNRs of the $L_{s}\left(\leq L_{c}\right)$ strongest paths among the $L_{a}$ paths from the target BS with the sum of the $L_{s}$ weakest SNRs among the currently used $L_{c}$ paths from the serving $\mathrm{BS}$, and selects the better group to form $L_{c}$ paths again. Note that the Block Change scheme just compares two blocks with equal size of $L_{s}$ and as such, avoids reordering all the paths which is essential for the Full GSC scheme. Therefore, a further reduction in SNR comparisons and SHO overhead can be obtained. The main contribution of this letter is to show a general comprehensive framework for the assessment of the Block Change scheme by providing analytical results for independent and identically distributed (i.i.d.) fading environments. More specifically, in our derivations we tackle the joint probability density function (PDF) of two adjacent partial sums of order statistics in order to obtain the statistics of the receiver output SNR.

\section{Finger Replacement-BAsed RAKE Combining}

\section{A. Channel and System Model}

Let $\gamma_{i}$ denote the instantaneous received SNR of the $i$ th resolvable path, $i=1,2, \cdots, L+L_{a}$. For analytical tractability, we assume first that the signals from all the resolvable 
paths experience i.i.d. Rayleigh fading conditions and the receiver operates over a "perfect" uniform power delay profile provided by a multi-path searcher in a way that the multipath components are correctly assigned to the RAKE fingers ${ }^{1}$. In this channel model, we do not consider the effect of inter-symbol/channel interferences by assuming, for example, perfect spreading codes. As such, the faded SNR, $\gamma_{i}$, follows the same exponential distribution with the common average faded SNR, $\bar{\gamma}$. We also assume that the pass-loss effects in the SHO region for different BSs are identical and therefore, we focus on the micro-diversity gain of the proposed scheme.

We consider the mobile unit which is equipped with an $L_{c}$ finger RAKE receiver and is capable of despreading signals from different BSs using different fingers, and thus facilitating the SHO process. More specifically, we assume that in the $\mathrm{SHO}$ region, the mobile unit is able to resolve the $L$ multipaths from the serving $\mathrm{BS}$ and the $L_{a}$ paths from the target $\mathrm{BS}$, and depending on the channel conditions only the $L_{c}$ paths among the $L$ or $L+L_{a}$ paths are used for RAKE reception according to the mode of operation described in the next section.

\section{B. Mode of Operation}

We focus on the receiver operation when the mobile unit is moving from the coverage area of its serving $\mathrm{BS}$ to that of a target BS. As the mobile unit enters the SHO region, the RAKE receiver relies at first on the $L$ resolvable paths gathered from the serving BS and as such starts with $L_{c} / L$ GSC. If we let $\Gamma_{i: j}$ be the sum of the $i$ largest SNRs among $j$ ones, i.e., $\Gamma_{i: j}=\sum_{k=1}^{i} \gamma_{k: j}$ where $\gamma_{k: j}$ is the $k$ th order statistics (see [5] for terminology), then the total received SNR after GSC is given by $\Gamma_{L_{c}}: L$. At the beginning of every time slot, the receiver compares the received $\mathrm{SNR}, \Gamma_{L_{c}}: L$, with a certain target SNR, denoted by $\gamma_{T}$. If $\Gamma_{L_{c}: L}$ is greater than or equal to $\gamma_{T}$, a one-way $\mathrm{SHO}$ is used and no finger replacement is needed. On the other hand, whenever $\Gamma_{L_{c}: L}$ falls below $\gamma_{T}$, a two-way SHO is attempted. In this case, the RAKE receiver compares the sums of two groups, the sum of the $L_{s}$ smallest paths among the $L_{c}$ currently used paths from the serving BS (i.e., $\sum_{i=L_{c}-L_{s}+1}^{L_{c}} \gamma_{i: L}$ ) and the sum of the $L_{s}$ strongest paths from the target BS (i.e., $\sum_{i=1}^{L_{s}} \gamma_{i: L_{a}}$ ). Then, the receiver replaces the $L_{s}$ smallest paths which are currently used with the best group. Note that no replacement occurs if the sum of the $L_{s}$ strongest additional paths from the target BS is less than the sum of the $L_{s}$ weakest paths among the $L_{c}$ strongest paths from the serving BS.

For simplicity, if we let

$$
\begin{gathered}
Y=\sum_{i=1}^{L_{c}-L_{s}} \gamma_{i: L}, \quad Z=\sum_{i=L_{c}-L_{s}+1}^{L_{c}} \gamma_{i: L}, \\
\text { and } W=\sum_{i=1}^{L_{s}} \gamma_{i: L_{a}}
\end{gathered}
$$

${ }^{1}$ In [6], more practical channel environments, such as nonidentical/correlated fading channels and outdated channel estimation, are considered. then, based on the above mode of operation, the final combined SNR, $\gamma_{t}$, is then given by

$$
\gamma_{t}= \begin{cases}Y+\max \{Z, W\}, & 0 \leq Y+Z<\gamma_{T} \\ Y+Z, & Y+Z \geq \gamma_{T} .\end{cases}
$$

\section{Statistics OF THE COMBINEd SNR}

In this section, we derive the statistics of the combined SNR of the proposed scheme.

From (1), the cumulative distribution function (CDF) of $\gamma_{t}$, $F_{\gamma_{t}}(x)$, can be written as

$$
\begin{aligned}
F_{\gamma_{t}}(x)= & \begin{cases}\operatorname{Pr}[Y+\max \{Z, W\}<x], & 0 \leq x<\gamma_{T} ; \\
\operatorname{Pr}\left[\gamma_{T} \leq Y+Z<x\right] & \\
+\operatorname{Pr}[Y+\max \{Z, W\}<x, & x \geq \gamma_{T} \\
\left.Y+Z<\gamma_{T}\right], & \end{cases} \\
= & \begin{cases}\operatorname{Pr}[Z \geq W, Y+Z<x] \\
+\operatorname{Pr}[Z<W, Y+W<x], & 0 \leq x<\gamma_{T} ; \\
\operatorname{Pr}\left[\gamma_{T} \leq Y+Z<x\right] & \\
+\operatorname{Pr}\left[Z \geq W, Y+Z<\gamma_{T}\right] \\
+\operatorname{Pr}[Z<W, Y+W<x, \\
\left.Y+Z<\gamma_{T}\right],\end{cases}
\end{aligned}
$$

Noting that $\operatorname{Pr}\left[\gamma_{T} \leq Y+Z<x\right]$ can be easily calculated from the CDF of $L_{c} / L$-GSC and that $W$ is independent of $Y$ and $Z$, we can obtain the other joint probabilities in (2) as

$$
\begin{aligned}
& \operatorname{Pr}[Z \geq W, Y+Z<x] \\
& =\int_{0}^{x} \int_{0}^{x-y} \int_{w}^{x-y} f_{Y, Z}(y, z) f_{W}(w) d z d w d y \\
& \operatorname{Pr}[Z<W, Y+W<x] \\
& =\int_{0}^{x} \int_{0}^{x-y} \int_{0}^{w} f_{Y, Z}(y, z) f_{W}(w) d z d w d y \\
& \operatorname{Pr}\left[Z \geq W, Y+Z<\gamma_{T}\right] \\
& =\int_{0}^{\gamma_{T}} \int_{0}^{\gamma_{T}-y} \int_{w}^{\gamma_{T}-y} f_{Y, Z}(y, z) f_{W}(w) d z d w d y \\
& \operatorname{Pr}\left[Z<W, Y+W<x, Y+Z<\gamma_{T}\right] \\
& =\int_{0}^{\gamma_{T}} \int_{0}^{x-y} \int_{0}^{\min \left\{w, \gamma_{T}-y\right\}} f_{Y, Z}(y, z) f_{W}(w) d z d w d y .
\end{aligned}
$$

Since $f_{W}(w)$ is the well-known PDF of $L_{s} / L_{a}$-GSC, we just need to find the joint PDF of $Y$ and $Z, f_{Y, Z}(y, z)$. For better illustration, let $l=L_{c}-L_{s}$ and $k=L_{c}$. Then, the order statistics of $L$ resolvable paths can be viewed as

$$
\begin{aligned}
& \underbrace{\overbrace{\gamma_{1: L}, \cdots, \gamma_{l-1: L}}^{A=\sum_{i=1}^{l-1} \gamma_{i: L}}, \gamma_{l: L}}_{Y=\sum_{i=1}^{l} \gamma_{i: L}}, \overbrace{Z=\sum_{i=l+1}^{k} \gamma_{i: L}}^{\overbrace{\gamma_{l+1: L}, \cdots, \gamma_{k-1: L}}^{B=\sum_{i=l+1}^{k-1} \gamma_{i: L}}, \gamma_{k: L}}, \gamma_{k+1: L}, \\
& \cdots, \gamma_{L: L}
\end{aligned}
$$

We start from the joint PDF of $A, \gamma_{l: L}, B$, and $\gamma_{k: L}$. Applying the Bayesian rule, we can write the joint PDF, $f_{A, \gamma_{l: L}, B, \gamma_{k: L}}(a, \alpha, b, \beta)$, as

$$
\begin{aligned}
& f_{A, \gamma_{l: L}, B, \gamma_{k: L}}(a, \alpha, b, \beta) \\
& =f_{\gamma_{l: L}}(\alpha) \cdot f_{\gamma_{k: L} \mid \gamma_{l: L}=\alpha}(\beta) \cdot f_{A \mid \gamma_{l: L}=\alpha, \gamma_{k: L}=\beta}(a) \\
& \quad \cdot f_{B \mid \gamma_{l: L}=\alpha, \gamma_{k: L}=\beta, A=a}(b) .
\end{aligned}
$$


After obtaining all PDFs in (8) (see [7] for detailed derivations), we can write a closed-form expression for the joint PDF, $f_{A, \gamma_{l: L}, B, \gamma_{k: L}}(a, \alpha, b, \beta)$, for i.i.d. Rayleigh fading channels as

$$
\begin{aligned}
& f_{A, \gamma_{l: L}, B, \gamma_{k: L}}(a, \alpha, b, \beta) \\
& =\frac{L ! e^{-(a+\alpha+b+\beta) / \bar{\gamma}}\left(1-e^{-\beta / \bar{\gamma}}\right)^{L-k}[a-(l-1) \alpha]^{l-2}}{(L-k) !(k-l-1) !(k-l-2) !(l-1) !(l-2) ! \bar{\gamma}^{k}} \\
& \quad \times \sum_{j=0}^{k-l-1}\left(\begin{array}{c}
k-l-1 \\
j
\end{array}\right)(-1)^{j} \\
& \quad \times[b-\beta(k-l-j-1)-\alpha j]^{k-l-2} \\
& \quad \times \mathcal{U}(\alpha) \mathcal{U}(\alpha-\beta) \mathcal{U}(a-(l-1) \alpha) \\
& \times \mathcal{U}(b-\beta(k-l-j-1)-\alpha j), \\
& \quad 0<(k-l-1) \beta<b<(k-l-1) \alpha .
\end{aligned}
$$

Since $Y=A+\gamma_{l: L}$ and $Z=B+\gamma_{k: L}$, we can obtain the joint pdf of $Y$ and $Z, f_{Y, Z}(y, z)$, from (9) by integrating out $\gamma_{l: L}$ and $\gamma_{k: L}$ as

$$
\begin{gathered}
f_{Y, Z}(y, z) \\
=\int_{0}^{\frac{z}{k-l}} \int_{\frac{z}{k-l}}^{\frac{y}{l}} f_{A, \gamma_{l: L}, B, \gamma_{k: L}}(y-\alpha, \alpha, z-\beta, \beta) d \alpha d \beta, \\
y>\frac{l}{k-l} z .
\end{gathered}
$$

Note that (10) involves only finite integrations of elementary functions and as such, this joint probability can be easily calculated with mathematical software, such as Mathematica. Also note that even though (10) is valid only when $l \geq 2$ and $k \geq l+2$, all other cases can be easily obtained by following the similar steps used for (9).

Finally, considering (10) together with (3)-(6), the CDF of $\gamma_{t}, F_{\gamma_{t}}(x)$, in (2) can be obtained.

By using the Leibnitz's rule [8, Eq. (6.40)] and differentiating (2) with respect to $x$, we can obtain after some manipulations the following generic expression for the PDF of the combined SNR, $\gamma_{t}$, as ${ }^{2}$

$$
\begin{aligned}
& f_{\gamma_{t}}(x) \\
& =\left\{\begin{array}{l}
\int_{0}^{x}\left(f_{Y, Z}(y, x-y) F_{W}(x-y)\right. \\
\left.+f_{W}(x-y) \int_{0}^{x-y} f_{Y, Z}(y, z) d z\right) d y, \quad 0 \leq x<\gamma_{T} ; \\
f_{Y+Z}(x) \\
+\int_{0}^{\gamma_{T}}\left(f_{W}(x-y) \int_{0}^{\gamma_{T}-y} f_{Y, Z}(y, z) d z\right) d y, x \geq \gamma_{T}
\end{array}\right.
\end{aligned}
$$

where $f_{Y, Z}(\cdot, \cdot)$ is defined in $(10), f_{W}(\cdot)$ and $f_{Y+Z}(\cdot)$ are the PDFs of $L_{s} / L_{a}$-GSC and $L_{c} / L$-GSC [9, Eq. (9.433)], respectively, and $F_{W}(\cdot)$ is the CDF of $L_{s} / L_{a}$-GSC [9, Eq. (9.440)].

\section{AVerage BER Performance}

In this section, we can apply the results from the previous section to analyze the average bit error rate (BER) performance of the proposed scheme by following the standard procedure presented in [9, Eq. (8.102)]. We now examine the

\footnotetext{
${ }^{2}$ To verify the correctness of our analysis, all the analytical results derived in this letter have been compared and verified by Monte Carlo Simulations.
}

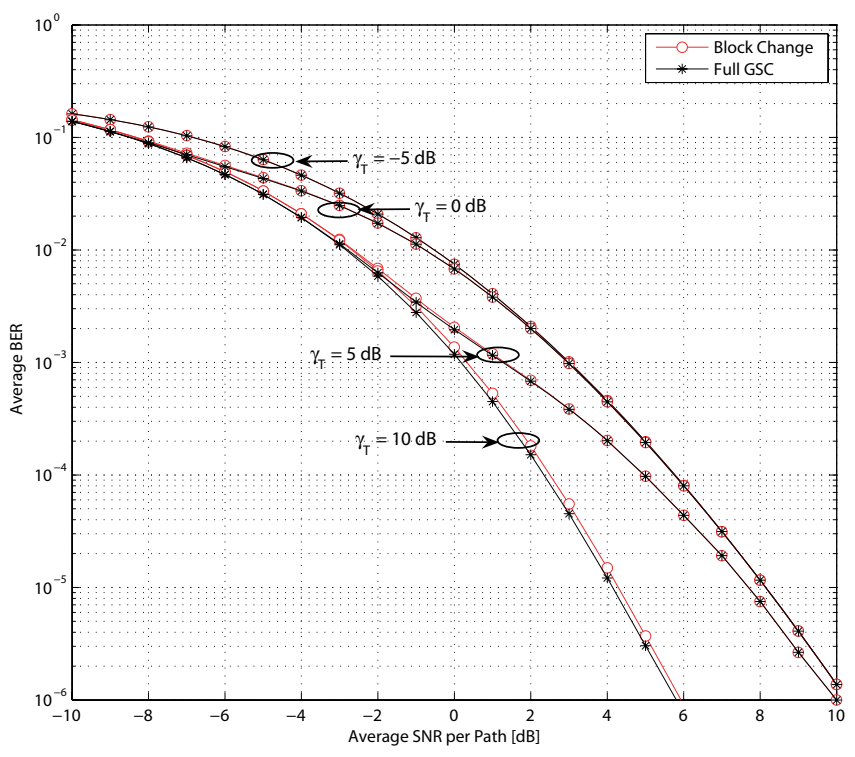

Fig. 1. Average BER of BPSK versus the average SNR per path, $\bar{\gamma}$, of the Block Change and the Full GSC schemes for various values of $\gamma_{T}$ over i.i.d. Rayleigh fading channels when $L=5, L_{a}=5, L_{c}=3$, and $L_{s}=2$.

BER performance of the Block Change scheme through two numerical examples.

In Fig. 1, we consider the effect of the switching threshold on the performance by representing the average BER of BPSK versus the average SNR per path, $\bar{\gamma}$, of the Block Change scheme proposed in this letter and the Full GSC scheme in [2] for various values of $\gamma_{T}$ over i.i.d. Rayleigh fading channels when $L=5, L_{a}=5, L_{c}=3$, and $L_{s}=2$. From this figure, it is clear that the higher the threshold, the better the performance, as we expect intuitively. Note that when $\bar{\gamma}$ becomes larger, the combined SNR is typically large enough in a way that the receiver does not need to rely on the additional paths from the target BS. Hence, we can observe that in good (1 h hannel conditions (i.e., $\bar{\gamma}$ is relatively large compared to $\gamma_{T}$ ), both schemes become insensitive to variations in $\gamma_{T}$. Also note that when the switching threshold is small, both schemes have almost the same performance since the additional paths are not necessary. On the other hand, in the case of large threshold values, the Full GSC scheme shows better performance since with the Full GSC scheme, instead of comparing and replacing blocks, the $L_{c}$ largest paths are selected among the $L+L_{a}$ ones.

In Fig. 2, we vary the block size, $L_{s}$, with two values of $\gamma_{T}$. We can see that for the low threshold, the variations of the block size do not affect the performance since in this case no replacement is needed. However, when the threshold is set high, we can observe the performance difference according to the value of $L_{s}$. For our chosen set of parameters, the best performance which is very close to that of the Full GSC scheme can be acquired when $L_{s}=2$. This is because if $L_{s}=1$, we have little benefit from the additional paths while if $L_{s}=3$, we have more chances to lose the better paths during the replacement process. 


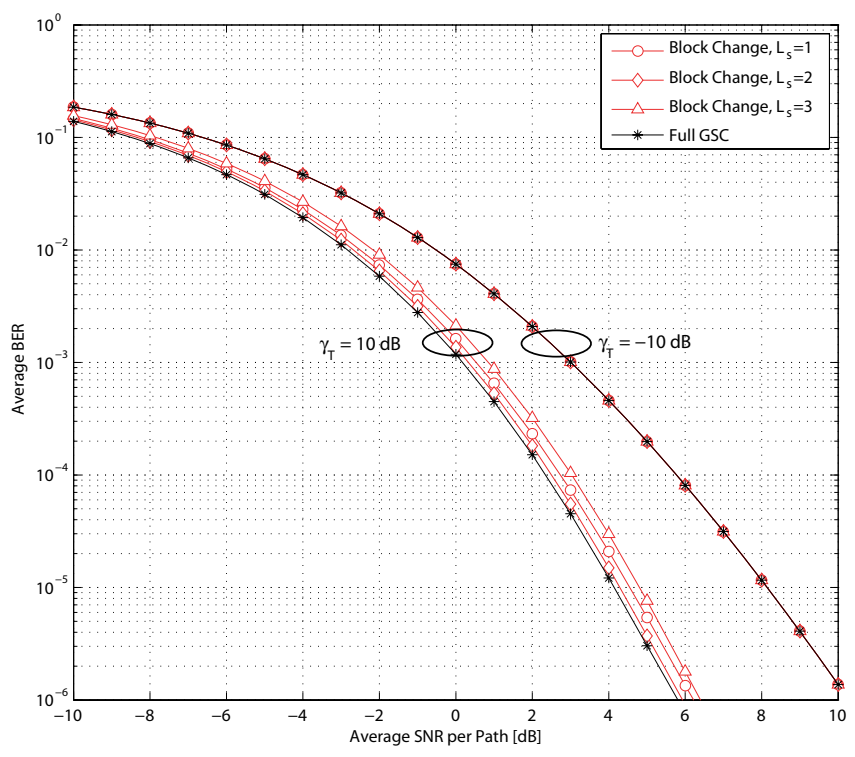

Fig. 2. Average BER of BPSK versus the average SNR per path, $\bar{\gamma}$, of the Block Change and the Full GSC schemes for various values of $L_{s}$ and $\gamma_{T}$ over i.i.d. Rayleigh fading channels when $L=5, L_{a}=5$ and $L_{c}=3$.

\section{COMPLEXITY COMPARISONS}

As shown in the previous section, because the paths with strongest SNR values are selected whenever needed, the Full GSC scheme always provides the better performance than the Block Change scheme. However, with the Block Change scheme considered in this letter, we can reduce the load induced by a full reordering process of all the paths at a slight sacrifice of performance. In this section, we investigate this complexity tradeoff issue by quantifying the average number of path estimations, the average number of SNR comparisons, and the SHO overhead.

\section{A. Average Number of Path Estimations}

With the proposed scheme, the RAKE receiver estimates the $L$ paths in the case of $\Gamma_{L_{c}: L} \geq \gamma_{T}$ or $L+L_{a}$ in the case of $\Gamma_{L_{c}: L}<\gamma_{T}$. Hence, the average number of path estimations of the Block Change scheme is same as that of the Full GSC scheme [2, Eq. (25)].

\section{B. Average Number of SNR Comparisons}

As another complexity measure, in this subsection we evaluate the average number of required SNR comparisons. Noting that the average number of SNR comparisons for $i / j$ GSC, denoted by $C_{G S C(i, j)}$, can be obtained as ${ }^{3}$

$$
C_{G S C(i, j)}=\sum_{k=1}^{\min [i, j-i]}(j-k),
$$

\footnotetext{
${ }^{3}$ With traditional sorting approach, we need $k-1$ comparisons to find the $k$ th largest/smallest one after the previous $k-1$ largest/smallest ones have been found. We follow this traditional approach in order to perform an accurate complexity comparison while noting that with quick sorting algorithm for $n$ paths, we just need $O(\log (n))$ complexity.
}

we can express the average number of SNR comparisons for the Full GSC scheme and the Block Change scheme as

$$
\begin{aligned}
C_{F u l l}= & \operatorname{Pr}\left[\Gamma_{L_{c}: L} \geq \gamma_{T}\right] C_{G S C\left(L_{c}, L\right)} \\
& +\operatorname{Pr}\left[\Gamma_{L_{c}: L}<\gamma_{T}\right] C_{G S C\left(L_{c}, L+L_{a}\right)}
\end{aligned}
$$

and

$$
\begin{aligned}
C_{\text {Block }}= & C_{G S C\left(L_{c}, L\right)}+\operatorname{Pr}\left[\Gamma_{L_{c}: L}<\gamma_{T}\right] \\
& \times\left(C_{G S C\left(L_{s}, L_{c}\right)}+C_{G S C\left(L_{s}, L_{a}\right)}+1\right)
\end{aligned}
$$

respectively.

\section{SHO Overhead}

In this section, we investigate the probability of the SHO attempt and the SHO overhead of the proposed scheme ${ }^{4}$. Since the SHO is attempted whenever $\Gamma_{L_{c}: L}$ is below $\gamma_{T}$, the probability of the $\mathrm{SHO}$ attempt is same as the outage probability of $L_{c} / L$-GSC evaluated at $\gamma_{T}$, i.e., $F_{\Gamma_{L_{c}: L}}\left(\gamma_{T}\right)$. The SHO overhead, denoted by $\beta$, is commonly used to quantify the SHO activity in a network and is defined as [10, Eq. (9.2)]

$$
\beta=\sum_{n=1}^{N} n P_{n}-1,
$$

where $N$ is the number of active BSs and $P_{n}$ is the average probability that the mobile unit uses $n$-way SHO. Based on the mode of operation in Section II-B, we can express the SHO overhead, $\beta$, of the Block Change scheme as

$$
= \begin{cases}F_{Y+Z}\left(\gamma_{T}\right) \operatorname{Pr}\left[Z<W \mid Y+Z<\gamma_{T}\right], & L_{s}<L_{c} \\ 0, & L_{s}=L_{c}\end{cases}
$$

Note that $F_{Y+Z}\left(\gamma_{T}\right)$ is the CDF of $L_{c} / L$-GSC output SNR [9, Eq. (9.440)] evaluated at $\gamma_{T}$. Since $W$ is independent to $Z$ and $Y$, we can calculate the conditional probability, $\operatorname{Pr}[Z<$ $\left.W \mid Y+Z<\gamma_{T}\right]$, in (16) as

$$
\begin{aligned}
& \operatorname{Pr}\left[Z<W \mid Y+Z<\gamma_{T}\right] \\
& =\int_{0}^{\infty} F_{Z \mid Y+Z<\gamma_{T}}(x) f_{W}(x) d x
\end{aligned}
$$

where

$$
\begin{aligned}
& F_{Z \mid Y+Z<\gamma_{T}}(x) \\
& =\frac{\operatorname{Pr}\left[Z<x, Y+Z<\gamma_{T}\right]}{\operatorname{Pr}\left[Y+Z<\gamma_{T}\right]} \\
& =\frac{1}{F_{Y+Z}\left(\gamma_{T}\right)} \times \\
& \begin{cases}\int_{0}^{x} \int_{l z /(k-l)}^{\gamma_{T}-z} f_{Y, Z}(y, z) d y d z, \\
\int_{0}^{(k-l) \gamma_{T} / k} \int_{l z /(k-l)}^{\gamma_{T}-z} f_{Y, Z}(y, z) d y d z, & x \geq \frac{k-l}{k} \gamma_{T},\end{cases}
\end{aligned}
$$

After successive substitutions from (18) to (16), we can finally obtain the SHO overhead.

In Fig. 3, we plot (a) the average number of SNR comparisons, (b) the average BER, and (c) the SHO overhead versus the output threshold, $\gamma_{T}$, of the Block Change and the Full

\footnotetext{
IV-C]
} 


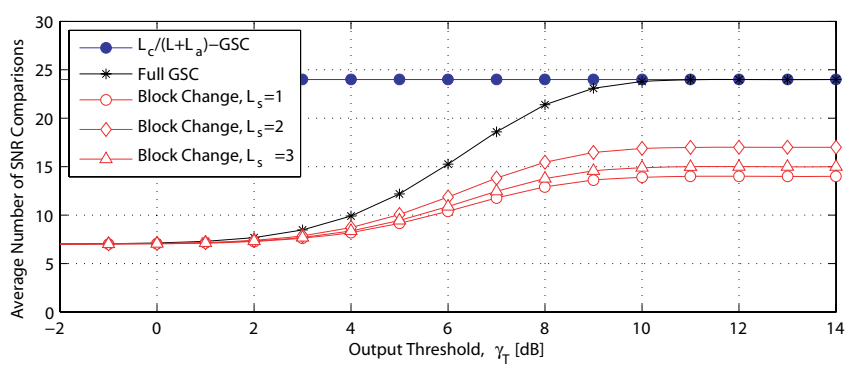

(a) Average Number of SNR Comparisons

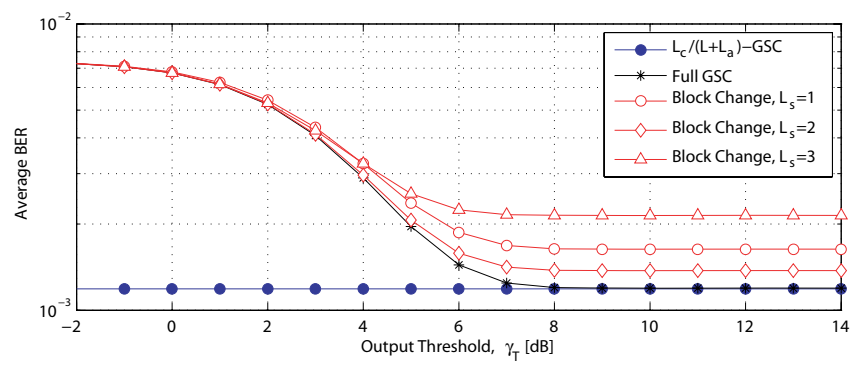

(b) Average BER

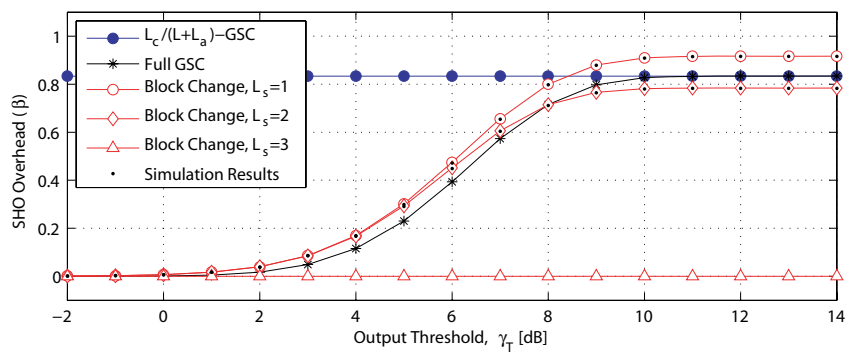

(c) SHO Overhead

Fig. 3. Complexity tradeoff versus the output threshold, $\gamma_{T}$, of the Block Change and the Full GSC schemes, and conventional GSC for various values of $L_{s}$ over i.i.d. Rayleigh fading channels with $L=5, L_{a}=5, L_{c}=3$, and $\bar{\gamma}=0 \mathrm{~dB}$.

GSC schemes for various values of $L_{s}$ over i.i.d. Rayleigh fading channels when $L=5, L_{a}=5, L_{c}=3$, and $\bar{\gamma}=0 \mathrm{~dB}$. For comparison purpose, we also plot those for conventional $L_{c} /\left(L+L_{a}\right)$-GSC. Note that the Full GSC scheme is acting as $L_{c} /\left(L+L_{a}\right)$-GSC when the output threshold becomes large. Hence, we can observe from all the sub-figures that the Full GSC scheme converges to GSC as $\gamma_{T}$ increases.

Recall that the Block Change scheme has the same path estimation load as the Full GSC scheme. However, from Fig. 3 (a), we can see that the Block Change scheme leads to a great reduction of the SNR comparison load compared to the Full GSC scheme. For example, let us consider the case that $\gamma_{T}>8 \mathrm{~dB}$ and $L_{s}=2$. In this case, the reduction of the SNR comparison load is maximized compared to the Full GSC scheme. However, from Fig. 3 (b), we can observe in the same SNR region a very slight performance loss of the
Block Change scheme compared to the Full GSC as well as the conventional GSC schemes.

For the SHO overhead, simulation results are also presented in Fig. 3 (c) to verify our analysis. It is clear from this figure that the receiver has a higher chance to use 2-way SHO as $L_{s}$ decreases. This is because as $L_{s}$ decreases, the probability that the sum of the $L_{s}$ smallest paths among the $L_{c}$ currently used paths from the serving BS is less than the sum of the $L_{s}$ strongest paths from the target BS is increasing and as such, we have a higher chance to replace groups. From this figure together with Fig. 3 (b), we can quantify the tradeoff between the SHO overhead and the performance. Again, let us consider the case that $\gamma_{T}>8 \mathrm{~dB}$ and $L_{s}=2$. In this case, note the reduction of $\mathrm{SHO}$ overhead at the expense of a slight performance loss in comparison to the Full GSC scheme. If we increase the number of fingers, i.e., $L_{c}=4$, we can observe more reductions in complexity with a slight performance loss. We omit these numerical results here due to space limitation.

\section{CONCLUSION}

In this letter, we proposed a new finger replacement scheme for RAKE reception in the SHO region. We provided a general comprehensive framework for the assessment of the proposed scheme by offering analytical results for i.i.d. fading environments. We showed through numerical examples that the proposed scheme can save a certain amount of complexity and SHO overhead with a very slight performance loss compared to the previously proposed scheme.

\section{REFERENCES}

[1] G. L. Stüber, Principles of Mobile Communication, 2nd ed. Norwell, MA: Kluwer Academic Publishers, 2001.

[2] S. Choi, M.-S. Alouini, K. A. Qaraqe, and H.-C. Yang, "Soft handover overhead reduction by RAKE reception with finger reassignment," IEEE Trans. Commun., vol. 56, no. 2, pp. 213-221, Feb. 2008.

[3] T. Eng, N. Kong, and L. B. Milstein, "Comparison of diversity combining techniques for Rayleigh-fading channels," IEEE Trans. Commun., vol. 44, no. 9, pp. 1117-1129, Sept. 1996.

[4] M. Z. Win and J. H. Winters, "Analysis of hybrid selection/maximalratio combining in Rayleigh fading," IEEE Trans. Commun., vol. 47, no. 12, pp. 1773-1776, Dec. 1999.

[5] M.-S. Alouini and M. K. Simon, "An MGF-based performance analysis of generalized selection combining over Rayleigh fading channels," IEEE Trans. Commun., vol. 48, no. 3, pp. 401-415, Mar. 2000.

[6] S. Choi, M.-S. Alouini, K. A. Qaraqe, H.-C. Yang, and Y.-C. Ko, "A practical study of adaptive finger reassignment and replacement in the soft handover region," in Proc. IEEE Int. Symp. on Signal Processing and its Applications (ISSPA'07), Sharjah, U.A.E., Feb. 2007.

[7] S. Choi, "Design and performance evaluation of RAKE finger management schemes in the soft handover region," Texas A\&M University, tech. rep. for Ph.D. dissertation.

[8] A. Papoulis and S. U. Pillai, Probability, Random Variables, and Stochastic Processes, 4th ed. New York: McGraw-Hill, 2002.

[9] M. K. Simon and M.-S. Alouini, Digital Communication over Fading Channels, 2nd ed. New York: John Wiley \& Sons, 2005.

[10] H. Holma and A. Toskala, WCDMA for UMTS, 3rd ed. New York: John Wiley \& Sons, 2004. 